\title{
Coronal holes in the long-term cosmic ray modulation
}

\section{Lev Dorman ${ }^{1}$}

Pushkov Institute of Terrestrial Magnetism, Ionosphere and Radio Wave Propagation, Russian Academy of Sciences, IZMIRAN, 142190, Troitsk, Moscow, Russia

Israel Cosmic Ray and Space Weather Center Institute of Advanced Study of Tel Aviv University, P.O. Box 39040, Tel Aviv, 69978, ISRAEL

E-mail: lid010529@gmail.com

\section{Anatoly Belov}

Pushkov Institute of Terrestrial Magnetism, Ionosphere and Radio Wave Propagation, Russian Academy of Sciences, IZMIRAN, 142190, Troitsk, Moscow, Russia

E-mail: abelov@izmiran.ru

\section{Raisa Gushchina}

Pushkov Institute of Terrestrial Magnetism, Ionosphere and Radio Wave Propagation, Russian Academy of Sciences, IZMIRAN, 142190, Troitsk, Moscow, Russia

E-mail: rgus@izmiran.ru

\section{Andrey Tlatov}

Kislovodsk Mountain Astronomical Station of Pulkovo Observatory

E-mail: tlatov@mail.ru

\section{Victor Yanke}

Pushkov Institute of Terrestrial Magnetism, Ionosphere and Radio Wave Propagation, Russian Academy of Sciences, IZMIRAN, 142190, Troitsk, Moscow, Russia

E-mail: yanke@izmiran.ru

The present study of galactic CR modulation in the heliosphere through the 21-24 cycles continues the series of works, where long-term CR modulation was described using the multiparametric model, including the solar activity (SA) characteristics. Initial data for modelling of $\mathrm{CR}$ variations are long-term observations of $\mathrm{CR}$ intensity, the characteristics of the solar global magnetic field and the short-time characteristic of SA (solar x-rays flares). Data of the CR intensity were obtained from the ground network of NM and stratospheric sounding. In order to improve the simulation of long-term $\mathrm{CR}$ variations we introduced into the model the characteristic of the regions with the open magnetic field - the coronal holes $(\mathrm{CH})$. Location (latitude), the area and the magnetic flux of $\mathrm{CHs}$ were used. Modeling modulation is carried out for all period and separately for the periods with the same polarity of the global field of the Sun, taking into account the delay $\mathrm{CR}$ variations regarding changes of SA characteristics. The quality of the long-term variations description has been improved by including in the model the $\mathrm{CH}$ characteristics.

The 34th International Cosmic Ray Conference

30 July- 6 August, 2015

The Hague, The Netherlands

${ }^{1}$ Speaker 


\section{Introduction}

The intensity of cosmic radiation observed on earth is the integral result of the modulation of cosmic rays (CR) by various solar and heliospherical phenomena. The connection between the observed flow of $\mathrm{CR}$ and cyclical solar activity has been confirmed by the results of observations of the Sun, of heliospherical characteristics and CR on earth and in space over many years. Modeling helps us to understand the modulation of $\mathrm{CR}$ by the electromagnetic fields of the heliosphere. Usually long-term CR variations are explained with the most popular index of solar activity - the sunspot number. However, for example, in the paper [1] it is proposed that the modulation of $\mathrm{CR}$ in the cycle of solar activity (SA) is influenced by changes in another unique, but more complex index - the solar non-axisymmetric (averaged in accordance with longitude) component of the open magnetic fluxes, which arises in active regions. However it is unlikely that just one parameter (solar or heliospherical) in fact controls CR behavior in the whole of the heliomagnetosphere.

The IZMIRAN group [2-5] proposes a semi-empirical, multi-parametric model which describes the long-term CR modulation (taking into account delays), basing the description mainly on the characteristics of the large-scale magnetic field of the Sun. Testing of many variants of the model, with different indexes and combinations of indexes showed that the best description of the quasi-cyclical long-term $\mathrm{CR}$ variations can be obtained by using a combination of the inclination of the heliospheric current sheet, the size of the magnetic field, averaged according to the surface of the source of solar wind and the size of the dipole component of the total magnetic field. In order to better assess the influence of sporadic solar phenomena a fourth index is added, reflecting the influence of CME on cosmic rays. This is possible only during the last two solar cycles [6]. For earlier and longer periods we have to find a substitute and use for example the flare index [5].The combination of these four solar indexes gives sufficient control of the long-term behavior of CR with a rigidity of $10 \mathrm{GV}$ on the Earth. This control is possible without taking into account the influence of coronal holes. This seems to be strange since the high speed fluxes of solar wind from coronal holes are an important part of the heliosphere. They have a significant influence on the interplanetary magnetic field, and their influence on CR is indisputable. Apart from this the speed of the solar wind determines two of the four main elements of the CR modulation mechanism: convection and adiabatic changes of energy particles. The influence of coronal holes is present in the most developed theoretical models of CR solar modulation, in some models it is the basis of the modulation [eg. 7].

There have been many attempts [eg. 8-12] to find the empirical links between the speed of solar wind near the Earth and the long-term changes in cosmic rays. It was possible to establish this link in some periods, but this research also showed that measurements of solar wind on the Earth are insufficient in order to draw any conclusions about the influence of coronal holes on cosmic rays throughout the entire heliosphere. It is necessary to develop alternative methods. For example measurements of interplanetary scintillation (IPS) make it possible to understand the long-term evolution of solar wind [13]. It appears that the evolution and migration of coronal holes, together with measurements of speed on Earth [14] and on the Sun on KA [15] can help in the study of the modulation effect of solar wind on CR.

An important obstacle to the study of the links between CR variation and coronal holes was the absence of good catalogues of coronal holes, catalogues which were complete and 
covered a sufficiently long period. Our work is based on a recent catalogue of coronal holes and on the papers [16, 17 and references therein], containing data on the area and magnetic flow of coronal holes observed from 1976 to 2012. Here we are expanding and improving the model of long term CR modulation [2-3] proposed earlier, which shows the success of our method. We are doing so by taking into account $\mathrm{CH}$ characteristics together with the earlier used characteristics of the Sun's magnetic field. The model covers 21-24 cycles of solar activity, for the whole period (1976-2012) and three periods with the same polarity of the global field of the Sun: $\mathrm{qA}<0$ of the negative $-1980-1990$ and 2000-2012 and $\mathrm{qA}>0$ of the positive 1990-2000.

\section{Data and method}

The model for research of CR modulation was developed in [2-5] and checked by the results of CR monitoring in the last five cycles of solar activity [18]. The original data for modeling of $\mathrm{CR}$ variations are the results of long-term observations of $\mathrm{CR}$ intensity, observations of the global magnetic field of the Sun, of X-ray flares (class $\geq$ M1) and the results of optical observations for the identification of coronal holes. All the data was organized on monthly base and were checked for quality. The CR variations in this paper were obtained by an earlier proposed method [19], where the isotropic part of cosmic radiation is determined on the basis of all the available information on the intensity of cosmic radiation registered in the worldwide network NM ( $\sim 40$ neutron monitors) and by probing the stratosphere in three places [20]. Further analysis was made with the value of CR isotropic variations with rigidity of $10 \mathrm{GV}$ (a10), which were obtained using this method, i.e. for particles for whose energy NM is most sensitive. We have excluded the variations connected with the Ground Level Enhancements of solar CR from the data and a10 is the long-term variation of galactic CR (in \% relative to 2009). In this way we can see the size of the variations of galactic origin, free from the influence of solar particles. As the structural and quantitative parameters of the global magnetic field of the Sun, calculated on the surface of the source of solar wind, we used the inclination of the heliosphere current sheet $\eta$, the average intensity of the of the magnetic field of the Sun Bss and the value of the polar field of the Sun with its sign Hpol. The choice of the parameters and the justification of the role of each of the above-mentioned indexes in the modulations of CR are set out in papers $[3,5,21]$. In order to take into account the modulation effect on $\mathrm{CR}$ of the sporadic activity of the Sun we propose the use of the specially calculated flare index Fx, defined in [5].

In this paper we have added the characteristics of coronal holes to the above-mentioned indexes of solar activity. The long-term cyclical evolution of coronal holes depending on their position, area and size of the magnetic flow has been studied in detail in [16-17]. For analysis we used the consolidated data of various observations of the areas and magnetic fluxes of coronal holes, shown in the paper [17], based on the results of observations at the observatory Kitt-Peak in the period 1975-2003 in the lines HeI $10830 \AA$ and SOHO/EIT in the lines FeXII $195 \AA$ in 1996-2012, and also SOLIS in the lines HeI $10830 \AA$ in 2004-2012. When we studied the link between coronal holes and CR we used data on their total area $\mathrm{S}$ and their total magnetic flux $\mathrm{F}$ (not taking into account negative or positive) and we divided the coronal holes according to latitude - low latitude from $\left(0^{\circ}-45^{\circ}\right)$ and high latitude $\left(45^{\circ}-90^{\circ}\right)$. As a result we obtained four indexes: Sl and Fl for low latitude CR and Sh and Fh for high latitude $\left(45^{\circ}-90^{\circ}\right)$. 

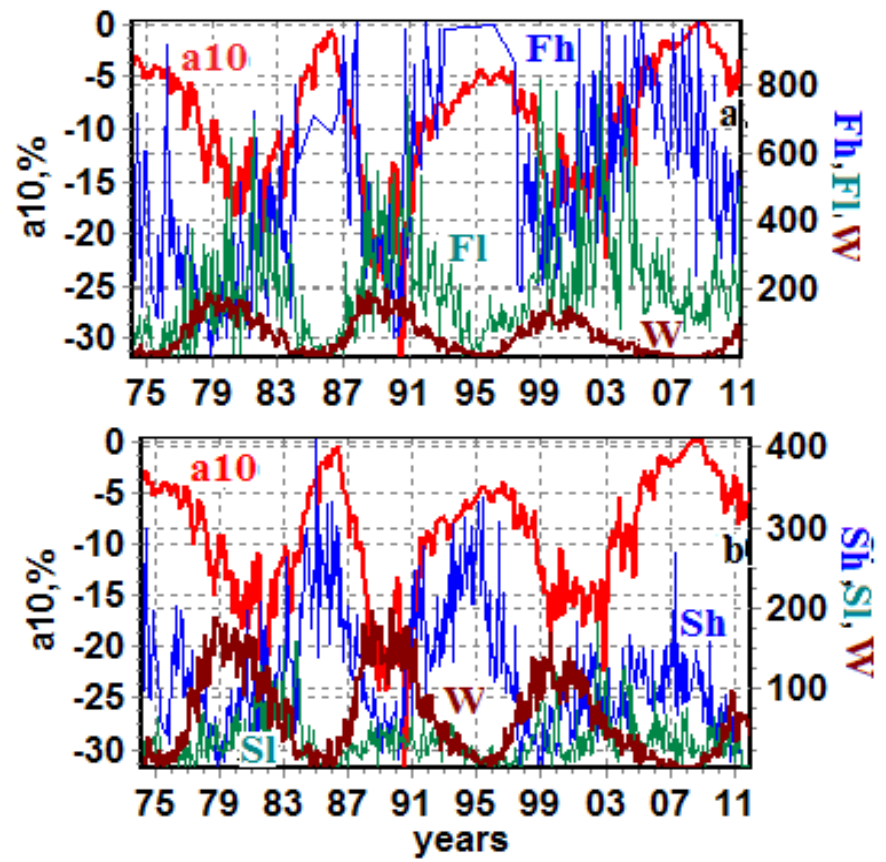

Fig.1. Temporary dependence of the amplitude of CR variations with a rigidity of $10 \mathrm{GV}$ (a10, \% to 2009) - left axis. The size of the magnetic flux of high latitude and low latitude holes $\mathrm{Fh}, \mathrm{Fl}$ (in units $10^{20} \mathrm{Mx}$ ) - right axis, upper panel, and area of coronal holes (low latitude $\mathrm{Sl}-3$ $10^{3}$ in millionths of the solar hemisphere area units (MSH), (high latitude $\mathrm{Sh}-10^{2} \mathrm{MSH}$ ), right axis, lower panel; W - number of solar spots.

The characteristics of coronal holes have an 11-year cycle (fig.1a,b). The phase of this cycle does not coincide with the well-known "classical" solar cycle, when the solar activity parameter is the sunspots $\mathrm{W}$ and correspondingly to with the intensity of CR - a10, the longterm variations of which are almost in counter phase with W (fig. 1a,b). If we look at the high latitude holes, at first glance it seems that the phase of their changes is nearer to the 11-year cycle of CR. Polar coronal holes appear after the polarity of the polar magnetic field changes. It is most prominent in a minimum of solar activity and can be observed until the next polarity reversal will occur. The low latitude coronal holes are situated in the zone of solar spots, the maximum of their cycle is shifted to the decline phase of the 11-year cycle of solar spots (correspondingly to the phase of the growth in the intensity of $\mathrm{CR}$, this was especially evident in the $21^{\text {st }}$ and $23^{\text {rd }}$ cycles of solar activity). The development of the cycle of low latitude coronal holes, just like the development of solar spots (the cycle of local fields), is shifted a half cycle in relation to the cycle of the large-scale field of the Sun.

Fig. 1 show that coronal holes have a peculiar behavior in the global cycles of the Sun, and indexes of activity connected with coronal holes do not repeat any of the other solar indexes.

\subsection{Model of CR modulations taking into account temporal and spatial changes of the coronal holes}

In this paper we (taking into account the changes of coronal holes (see fig.1a,b) have modeled CR modulations for the period 06.1976 - 12.2012 and for the periods with a certain polarity of the Sun's global field: negative values of the polarity of the Sun's global field qA $<0$ (01.1980- 
01.1990 and $03.2000-12.2012)$ and positive $\mathrm{qA}>0$ (02.1990-02.2000). We used the description of the long-term modulation of CR with the aid of a tested model with the four solar indexes (including the above-mentioned indexes $\eta$, Bss, Fx and one of the coronal hole indexes (area or the absolute magnitude of the magnetic flux). In order to take into account the modulators effect on CR intensity of the index Hpol, we made an adjustment in the amplitude of the variation a10. This was determined from the results of the model description of CR large-scale modulation using regression equations for the SA parameters ( $\eta$, Bss, Fx and Hpol).

After the implementation of this adjustment of the amplitude (a10) we carried out a multiparametric linear approximation of CR variation for 1976-2012, taking into account the combined influence on CR of the following parameters of SA: $\eta$, Bss, Fx, adding to each in turn one of the coronal hole characteristics: Sh, Fh, Sl and Fl.

In this way, in our model of CR modulations, the magnitude of the observed CR modulations is determined by the influence on $\mathrm{CR}$ of one of the coronal hole characteristics (by area or magnetic flux, taking into account the latitudinal location of the holes) and the abovementioned solar indexes ( $\eta$, Bss, xf and Hpol).
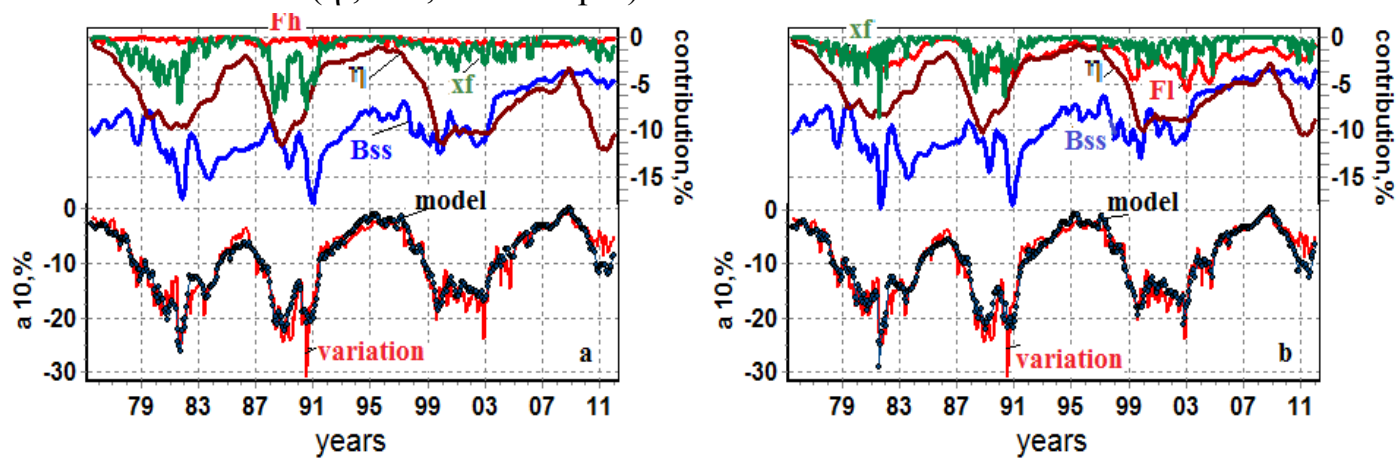

Fig.2. a10 - CR variations (in \% to 2009) observed and calculated according to model (lower part); contribution (\%) of changes: a) xf, Fh, Bss, $\eta$ and from changes b) xf, Fl, Bss, $\eta$ in expected CR variations for 1976-2012 (upper part)

As a result, when we take additional account of each parameter which characterizes change in area or magnetic flux of coronal holes, we see an improvement in the model of CR modulation with a relatively small difference of the coefficients of the correlations $p$ for this period. The highest value of $\rho=0.95$ (the mean square error $\sigma=2.0 \%$ ) received for $\mathrm{Fl}$ of the magnetic flux of low latitude coronal holes. From fig. 2 a,b we can see that the contribution of coronal holes to $\mathrm{CR}$ modulation from this parameter of solar activity during the whole of the analyzed period could be $>6 \%$, while the contribution of the flux Fh from high latitude holes does not exceed $2 \%$, and most of the time it is $<0.5 \%$.

Analogical approximation, carried out for the coronal hole areas (indexes Sh and Sl) yielded similar results, but with a slightly bigger difference between the modeled and observed variations.

In order to study the dependence of CR modulations on the changes in area and magnetic flux of coronal holes for the periods with the same polarity of the Sun's global field, we conducted model calculations for intervals from the solar maximum of one cycle to the solar maximum of the next: for the negative polarity of the field $\mathrm{qA}>0-01.1980-01.1990$ and 03.2000-02.2012 and positive $\mathrm{qA}<0-02.1990-02.2000$. 

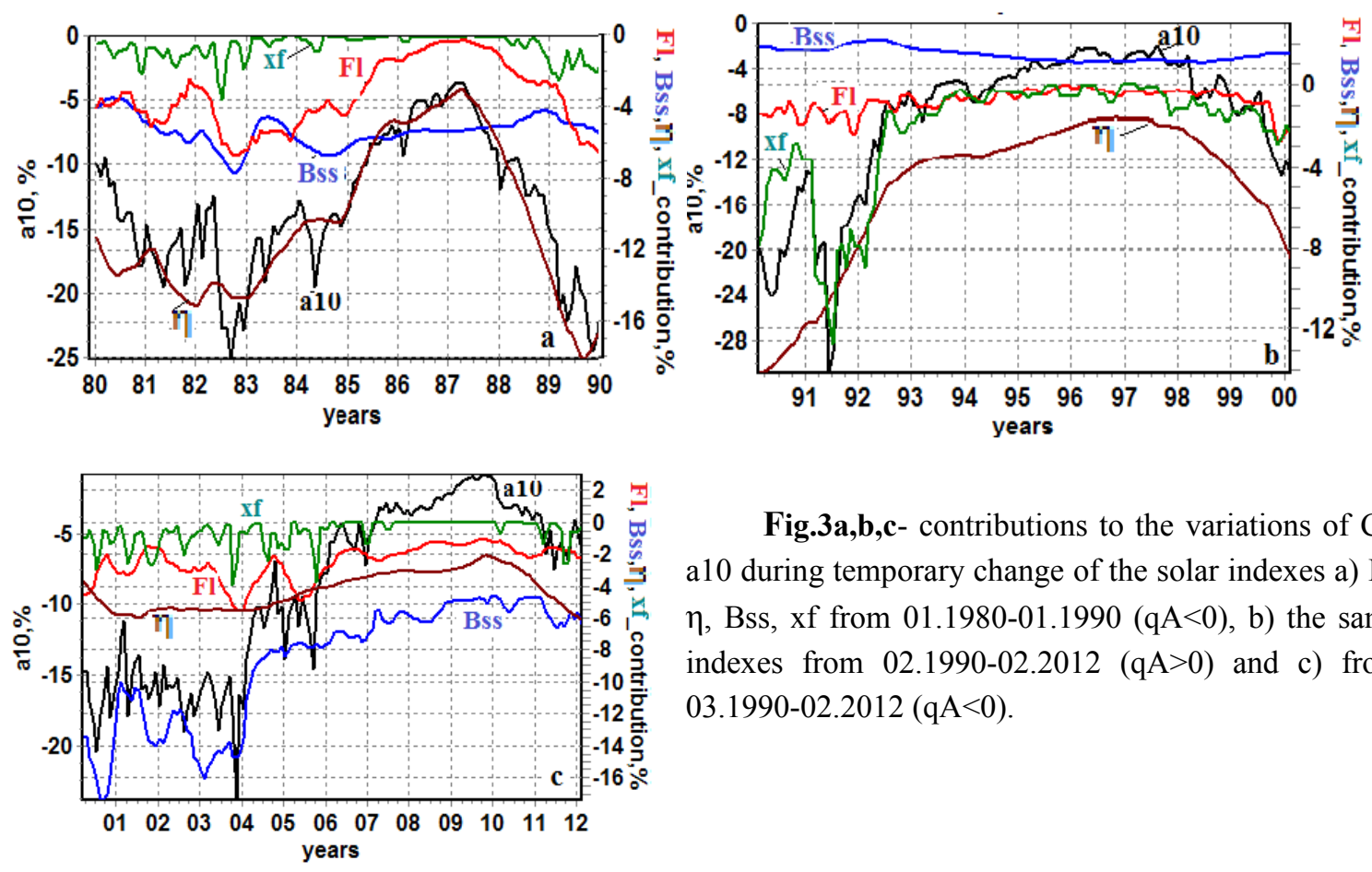

Fig.3a,b,c- contributions to the variations of CR a10 during temporary change of the solar indexes a) Fl, $\eta$, Bss, xf from 01.1980-01.1990 (qA<0), b) the same indexes from 02.1990-02.2012 (qA>0) and $\mathrm{c})$ from 03.1990-02.2012 (qA<0).

In fig. $3 \mathrm{a}, \mathrm{b}, \mathrm{c}$ for the three periods we show the contributions to the $\mathrm{CR}$ variations from the changes of the model solar parameters. The changes in the magnetic flux Fl in the cycles with negative polarity of the global magnetic field of the Sun (1980-1990 and 2000-2012) have a value of $>6 \%$. They are close to the changes in the inclination of the current sheet $\eta$, but fairly original. As we can see in fig. $3 \mathrm{~b}$, the changes in the magnetic flux Fl are completely different for other solar magnetic field polarity. In this case the contribution to $\mathrm{CR}$ variations is significantly less, and less noticeable in the 11-year cycle.
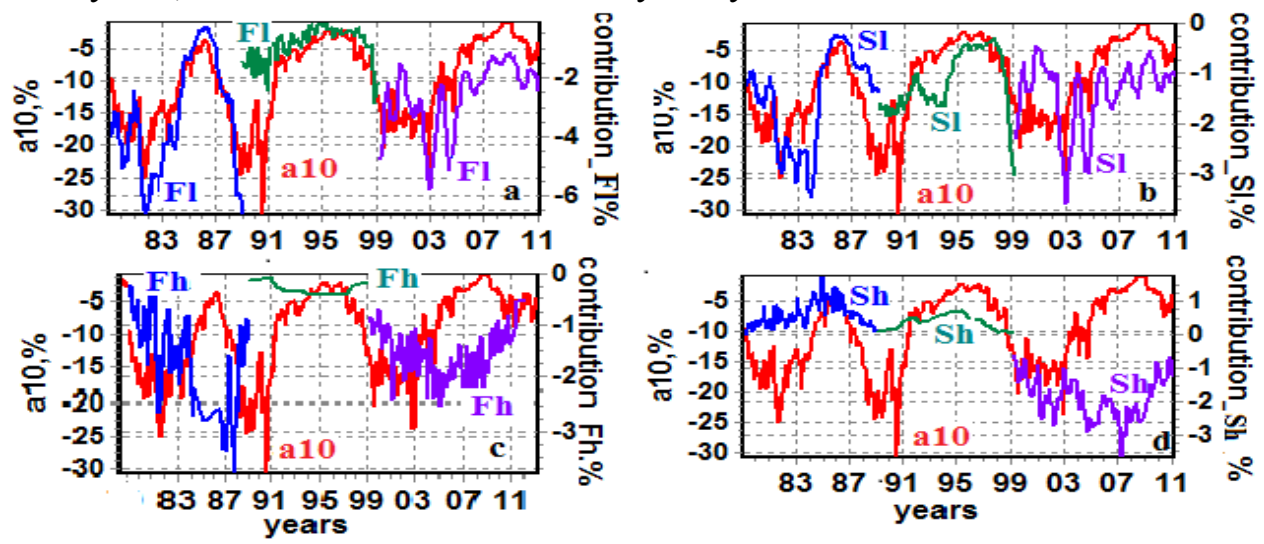

Fig.4a,b,c,d. Observed $C R$ variations (a10) and the calculated contribution to $C R$ modulation of the changes in coronal hole area (b, $d$ low latitude and high latitude) and of the coronal hole magnetic fluxes (a, c low latitude and high latitude) in 1980- 2012.

In fig. 4 (a-d) we have combined the CR variations (a10, \%) for 1980-2012 and contributions to the observed variation of the changes in coronal characteristics for all three periods shown. It is clear that the largest contribution to modulation is from the magnetic flux Fl of the low latitude holes $(>6 \%)$ and this contribution change in phase with $\mathrm{CR}$ variations (in solar minimums contributions to CR modulation are small). The degree of CR modulations changes depending 
on the polarity of the Sun's global magnetic field due to the low latitude holes: for $\mathrm{qA}<0$ it is 3$6 \%$, and for $\mathrm{qA}>0$ it lessens to $\sim 1-2 \%$. We should note that the modulation influence of the magnetic flux of the high latitude holes on CR intensity is significantly less than the contribution of low latitude holes - for $\mathrm{qA}<0$ it is up to $3 \%$ and for $\mathrm{qA}>0$ only $\sim 0.5 \%$.

For the areas of low latitude holes the greatest contribution is observed for $\mathrm{qA}<0$ and is $3 \%$ (fig. 4b). As for the CR modulations connected with change in the areas of high latitude coronal holes, in the 1980s and 1990s the influence of this characteristic resulted in a decrease of modulation (to $1 \%$ ) and only in the $23^{\text {rd }}$ extraordinary cycle was there a significant effect on $\mathrm{CR}$ modulation when the coronal hole area was $\mathrm{qA}<0$ (fig.4d).

We can conclude that the coronal holes magnetic flux from low latitude holes Fl makes a significant contribution to $\mathrm{CR}$ modulation, which by far exceeds the contribution from high latitude coronal holes Fh. The contribution of the coronal hole indexes for the period $\mathrm{qA}<0$ (1980-1990) is noticeably greater than during $\mathrm{qA}>0$ period 1990-2012. Regarding the contribution of coronal hole area we can say that the influence on CR modulation of low latitude holes $\mathrm{Sl}$ is similar on the flux Fl, but with a slightly smaller contribution. However, the area of high latitude coronal holes only in the cycle with a negative polarity of the Sun's global field in 1990-2012, the so-called "anomalous cycle" had a significant influence on CR modulation.

It is important to note that low latitude and high latitude coronal holes have an opposite influence on CR modulation. The influence of high latitude holes is mainly in counter-phase to the other modulator indexes. The low latitude holes intensify modulation whereas the high latitude holes diminish modulation. This is possible to explain qualitatively. The bigger the coronal holes in the low heliolatitudes the greater the regions of interaction of the fluxes of solar wind with different speeds (GMIR) in which the interplanetary field becomes stronger and prevents CR penetration into the inner heliosphere. Above the polar holes the IMF structure is more simple. There are no GMIRs and no other reasons for a significant increase in IMF. For this reason it is easier for galactic cosmic rays to penetrate these zones of the heliosphere. If we imagined a Sun covered by two polar coronal holes, divided by a flat current sheet we would have a heliosphere with far weaker CR modulation than in our real heliosphere.

This analysis shows that when studying CR variations it is not advisable to mix high lattude and low latitude holes. If we had combined all the holes, without regard for their helio latitude, into common indexes, the results would be significantly inferior.

\section{Main conclusions}

We have presented modeling of long-term CR variations in the period 1976-2012 based on the characteristics of the Sun's general magnetic field, with the addition of indexes that characterize coronal holes. The use of the total (for each month) area of the holes and the total magnetic flux from them yielded similar results with a slight advantage of the magnetic flux.

It is shown that low latitude coronal holes have a significant influence on galactic CR modulation and the addition of the relevant indexes has noticeably improved the model. Indexes characterizing high latitude holes have a significantly weaker influence on $\mathrm{CR}$ variations.

The influence of coronal holes on CR modulation is noticeably stronger when the polarity of the general magnetic field is negative, and weaker when it is positive. The influence of high and low latitude coronal holes is quite contradictory. The low latitude holes intensify modulation whereas the high latitude holes diminish it. 


\section{Acknowledgments}

This work is partly supported by Program № 10 BR of the Presidium RAS “The fundamental properties of matter and Astrophysics", Program № 22 BR of the Presidium RAS "Fundamental processes of research and development of the Solar System". A.T. would like to acknowledge partial support from the Russian Science Foundation grant No. 15-12-20001. We thank all the members of the network stations of cosmic rays http://cr0 inran.ru/ThankYou

\section{References}

1. Y.-M. Wang, N. R. Sheeley, Jr., A. P. Rouillard, ApJ., 644, 638 (2006).

2. A.V. Belov et al., JASTP, 63, 18, 1923, (2001).

3. A.V. Belov et al., J.Geomagn. Aeron., 42, 639 (2002).

4. A.V. Belov et al., ASR, 35, 3, 491 (2005).

5. A.V. Belov et al, Bull. Russ. Acad. Sci.: Phys, 71, No. 7, 974 (2007).

6. E. Paouris et al, Solar Phys., 280. 1, 255 (2012).

7. G.F. Krymsky et al, Bull.Russ. Acad. Sci.: Phys., 65, N 3, 353 (2001).

8. S.P.Duggal, M.A. Pomerantz, 15th ICRC, 221 (1977).

9. R.L.Sigh et al, 18th ICRC, 3, 225 (1983).

10. N.P. Chirkov, J.Geomagn. Aeron., 19, N2, 217 (1979).

11. N.P. Chirkov, 19th ICRC, 4, 489 (1985).

12. A.V.Belov et al, Bull. Russ. Acad. Sci.: Phys, 65, N3, 360 (2001).

13. P.K. Manohara, http://arxiv.org/astro-ph/arXiv1203.6715v1 (2012).

14. W.A. Coles, B.J Rickett, Solar-Geophysical Data (1973-1981).

15. McComas et al, Geophysical Res Lett, 35, L18103 (2008).

16. K.S. Tavastsherna, A.G.. Tlatov, Catalog and atlas of synoptic charts coronal holes and cavities of the filaments in the line He I 10830 A. The period 1975 - 2003. Pulkovo Astronomical Observatory RAS, St. Petersburg (2006).

17. A. Tlatov, K. Tavastsherna and V. Vasil'eva, Solar Physics, 289, 1349, DOI:10. 1007/s11207-0130387-4.

18. R.T. Gushchina et al., Geomagn. Aeron., 52, N4, 438 (2012).DOI: 10.1134/S0016793212040068

19. A.V. Belov, R.T. Gushchina and I.V. Sirotina, 23rd ICRC, 3, 605 (1993).

20. Yu.I. Stozhkov et al, Cosmic ray fluxes at an absorption curve maximum in the atmosphere and at the atmospheric boundary (1957-2007), Preprint, RAS, Moscow, 55 p. (2007).

21. R.T. Gushchina et al, J.Geomagn. Aeron., 48, 571 (2008) 